\title{
PRONÓSTICO Y ANÁLISIS DEL IMPACTO DEL FENÓMENO EL NIÑO EN EL NORTE DEL PERÚ
}

\author{
Inés Gambini Lopez ${ }^{1}$, Esther Berger Vidal', \\ José Carlos Oré Luján ${ }^{3}$ \& Luis Pizarro Pereyra ${ }^{4}$
}

\begin{abstract}
Resumen: El Niño es uno de los fenómenos océano-atmosféricos que con mayor frecuencia impacta la región norte del Perú, se caracteriza por producir intensas lluvias en la zona norte del Perú, causando enormes pérdidas económicas y daños en la agricultura, infraestructura vial, pesquería, en el sector educación, sector salud, entre otros. Basados en la información histórica mensual de la temperatura superficial del mar de las zonas de Tumbes, Paita y Chicama y utilizando las herramientas estocásticas de predicción como son las Cadenas de Markov, en esta investigación se pretende pronosticar la posible aparición de estos eventos frente ạl mar peruano, de acuerdo a diferentes niveles de intensidad. Utilizando la serie de datos mensuales disponible para cada puerto hasta el año 2008 y en base a una matriz de transición de una cadena de Markov se hace un pronóstico de la posible aparición de un evento El Niño en el año 2009 de acuerdo a escalas de intensidad para las zonas de Tumbes, Paita y Chicama en una matriz de probabilidades de transición.
\end{abstract}

Palabras clave: Cadenas de Markov, Matriz de transición, Temperatura superficial del mar

\section{FORECAST AND ANALYSIS OF THE NIÑO PHENOMENON OVER THE NORTH OF PERU}

\begin{abstract}
El Niño is one of the phenomena ocean atmospheric that with major frequency impacts over the north region of Peru, which is characterized for intensity rains in the north zone of Peru, causing enormous economic losses and damages in the agriculture, road infrastructure, fisheries, in the education sector, health sector between others. Based on the historical monthly information of the sea surface temperature of the zones Tumbes, Paita and Chicama and using the tools stochastic of prediction as like The Markov's chains, in this research one tries to predict the possible appearance of these events front the Peruvian sea in agreement to different levels of intensity. Using the series of monthly information from beginning until the year 2007 and on the basis of a matrix of transition of Markov's chain, there becomes an attempt of forecast of the appearance of an event El Niño for 2009 of agreement to scales of intensity for the zones of Tumbes, Paita and Chicama in a transitional probabilities matrix.
\end{abstract}

Key words: Markov's chains, transition matrix, Sea surface temperature

\footnotetext{
${ }^{1}$ Departamento Académico de Investigación Operativa. UNMSM, e-mail: inesgambinil@hotmail.com

${ }^{2}$ Departamento Académico de Investigación Operativa. UNMSM, e-mail: esther_berger_v@yahoo.es

${ }^{3}$ Departamento Académico de Investigación Operativa. UNMSM, e-mail: ore240@hotmail.com

${ }^{4}$ Instituto del Mar del Perú, e-mail: lpizarro@imarpe.gob.pe
} 


\section{Introducción}

La mayor frecuencia de aparición del Fenómeno El Niño en la costa del Perú, afecta al medio ambiente produciendo sequías y lluvias abundantes en diferentes lugares del país, lluvias intensas en la costa, friaje y sequía en los Andes, provocando pérdida de cosechas, animales, viviendas y puestos de trabajo, así como la migración de las poblaciones afectadas. El objetivo del presente estudio es pronosticar la posible aparición del Fenómeno El Niño de acuerdo a la información histórica, por niveles de intensidad, los cuales van desde Niño débil a moderado, intenso y extraordinario de acuerdo a las temperaturas sobre el nivel del mar y el impacto que produce su aparición en las zonas críticas del país.

Considerando que los tipos de Niño son estados posibles de un proceso estocástico, se ha enfocado este fenómeno como una Cadena de Markov en la cual se definen las transiciones posibles entre estos estados. Se dispone de registros de las temperaturas superficiales marinas en un puerto del norte del país a lo largo del tiempo, sobre los cuales se hace un estudio piloto para realizar predicciones de la aparición y del posible impacto en esa zona, tanto en la Costa como en los Andes y en la Amazonía, de acuerdo al tipo de fenómeno, mediante el análisis de la cadena de Markov. Este estudio piloto brinda la metodología para el análisis futuro en otras zonas costeras de la costa central y de la costa sur, observándose el impacto en las zonas transversales correspondientes. Se realizará un mapeo de los efectos del fenómeno a fin de tomar las medidas de solución para prevenirlos y para contrarrestarlos cuando ocurran.

Los eventos El Niño son un fenómeno climático cíclico que provoca estragos a nivel mundial, siendo las zonas más afectadas América del Sur y la zona entre Indonesia y Australia, provocando con ello el calentamiento de las aguas Sudamericanas.

Su nombre se refiere al niño Jesús, porque la ocurrencia del fenómeno se da aproximadamente en mes de diciembre que es el tiempo de Navidad en el Océano Pacífico, en la costa Oeste del Sur de América. Es un síndrome con más de 7 milenios de ocurrencia.

En el océano Pacífico tropical los eventos "El Niño" son detectados mediante diferentes métodos, que van desde satélites y boyas flotantes hasta análisis del nivel del mar, obteniendo importantes datos sobre las condiciones en la superficie del océano. Por ejemplo, las boyas miden·la temperatura, las corrientes y los vientos en la banda ecuatorial, toda esta información la transmiten a los investigadores de todo el mundo.

La mayor frecuencia de aparición del Fenómeno El Niño en la costa del Perú, afecta al medio ambiente produciendo sequías y lluvias abundantes en diferentes lugares del país, lluvias intensas en la costa, friaje y sequía en los Andes e inundaciones en la Amazonía, provocando pérdida de cosechas, animales, viviendas y puestos de trabajo, así como la migración de las poblaciones afectadas.

\section{Objetivo}

El estudio que se ha realizado en los puertos de Tumbes, Paita y Chicama, tiene la finalidad de hacer pronósticos para el año 2009 sobre el tipo de fenómeno El Niño que se pudiera presentar y cuál sería el impacto en la economía del país en especial en el sector pesquero debido a que la pesca de la anchoveta genera altas divisas para el Perú.

\section{Metodología}

El presente estudio trata de las diferentes temperaturas promedio superficiales de los puertos de las aguas marinas registradas en Tumbes, Paita y Chicama que han sido proporcionadas por el 
Instituto del Mar del Perú (IMARPE ) las cuales son medidas diariamente por 5 a 7 equipos, que se encuentran instalados en cada puerto.

Los pasos a seguir son los siguientes:

1. Se clasifica la temperatura superficial del mar en diferentes estados de acuerdo a los eventos que se dan por rangós de temperatura, construyendo la matriz de transición de las cadenas de Markov y haciendo los análisis respectivos de proyección a futuro de $P_{n}$ para el año 2009.

2. Se define el conjunto de posibles estados $\mathbf{E}$, así como el espacio paramétrico $T$, los cuales caracterizan la situación en que se halla el sistema en un instante dado

3. La definición de transición como el cambio del valor de la variable temperatura en el tiempo. Estos posibles valores de $E$ se toman de un conjunto de categorías mutuamente excluyentes.

4. Una ley de probabilidad condicional. Se puede expresar mediante la matriz de probabilidades de transición $\mathbf{P}$, matriz de la cadena de Markov. La matriz $P$ es una matriz $m \times m$, donde $\mathrm{m}$ es el número de estados del sistema y los elementos de la matriz representan la probabilidad de pasar del estado $i$ al estado $j$ en un paso, con $i$ estado fila y $j$ estado columna.

La probabilidad de transición debe cumplir la siguiente propiedad:

$$
\sum_{j=1}^{n} p_{i j}=1, \text { donde } p_{i j} \geq 0
$$

La matriz $P$ es una matriz estocástica : la suma de valores de las filas de la matriz será igual a uno.

Considerando que los tipos de Niño son estados posibles, se considera en este estudio un proceso estocástico, en particular una Cadena de Markov en la cual se definen las transiciones posibles entre estos estados. Esta cadena cumple con:

1. La Propiedad Markoviana.

2. La Homogeneidad de las probabilidades.

3. El Espacio de Estados es Discreto.

4. El Espacio Paramétrico es Discreto.

En la primera parte el estudio trata acerca de las diferentes temperaturas promedio superficiales de las aguas marinas registradas en el puerto de Chicama entre los años 1925 y 1996, Clasificando los diferentes estados y construyendo la matriz de transición de las cadenas de Markov y haciendo los análisis respectivos de proyección a futuro de las probabilidades dadas en la matriz de transición $P_{1}$ para el año 1997.

Definimos los Estados:

$\begin{array}{llll}\text { 1. Niño Extraordinario } & \text { NE } & \text { 5. Año Normal } & \text { N } \\ \text { 2. Niño Fuerte } & \text { NF } & \text { 6. Año Frío } & \text { F } \\ \text { 3. Niño Moderado } & \text { NM } & \text { 7. Muy Frío } & \text { MF } \\ \text { 4. Niño Débil } & \text { ND } & \text { 7. Mur }\end{array}$

Donde $E=\{1,2,3,4,5,6,7\}$ que es la interpretación numérica de esta clasificación. Se define el espacio Paramétrico:

$$
\mathrm{T}=\{1925,1926,1927, \ldots, 1996\}
$$

La matriz que se presenta corresponde a los años 1925 hasta 1996 siendo en total 71 años, Estimamos las probabilidades de transición de una Cadena de Markov de la siguiente manera:

donde $\mathbf{N}_{\mathbf{i}}=$ frecuencias en que aparecen los estados definidos de un período a otro.

Asociada a la Cadena de Markov se tiene la Matriz de Probabilidades de Transición $P$ 


\begin{tabular}{ll}
\hline \multirow{2}{*}{ NOMBRES } & $\begin{array}{l}\text { TEMPERATURA OBSERVADA } \\
\text {-VALOR PROMEDIO (UT) }\end{array}$ \\
\hline NIÑO EXTRAORDINARIO & $>+2.7^{\circ} \mathrm{C}$ \\
NIÑO FUERTE & $+1.7+2.7^{\circ} \mathrm{C}$ \\
NIÑO MODERADO & $+0.8+1.6^{\circ} \mathrm{C}$ \\
NIÑO DÉBIL & $+0.5+0.7^{\circ} \mathrm{C}$ \\
AÑO NORMAL & $-0.6+0.4^{\circ} \mathrm{C}$ \\
AÑO FRÍO & $\leq-0.7^{\circ} \mathrm{C}$ \\
MUY FRÍO & $\leq-1.8$ \\
\hline
\end{tabular}

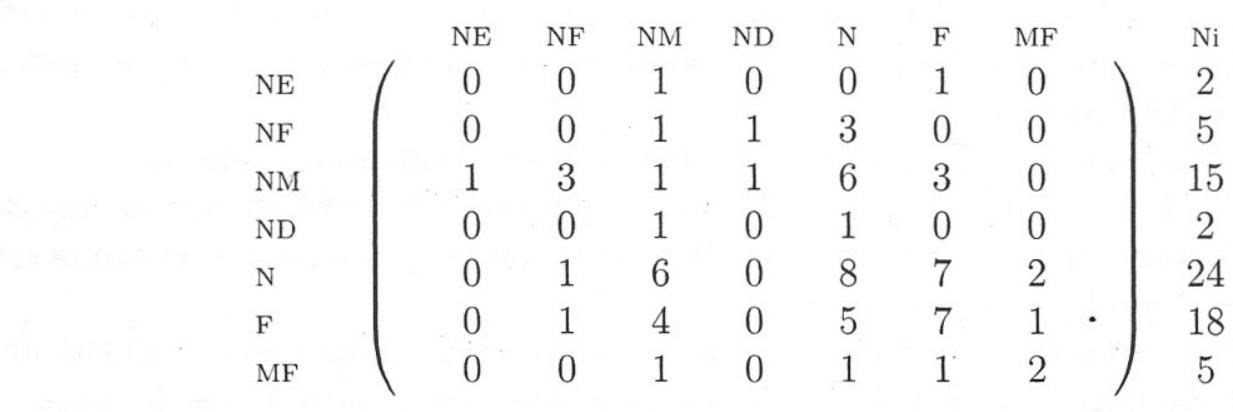

\begin{tabular}{|c|c|c|c|c|c|c|c|c|}
\hline & & & & & $X_{n+1}$ & & & \\
\hline & & $\mathrm{NE}$ & $\mathrm{NF}$ & NM & ND & $\mathrm{N}$ & $\mathrm{F}$ & MF \\
\hline & $\mathrm{NE}$ & 0 & 0 & $1 / 2$ & 0 & 0 & $1 / 2$ & 0 \\
\hline & $\mathrm{NF}$ & 0 & 0 & $1 / 5$ & $1 / 5$ & $3 / 5$ & 0 & 0 \\
\hline & $\mathrm{NM}$ & $1 / 15$ & $3 / 15$ & $1 / 15$ & $1 / 15$ & $6 / 15$ & $3 / 15$ & 0 \\
\hline $\mathbf{P}=\mathbf{X}_{\mathbf{n}}$ & ND & 0 & 0 & $1 / 2$ & 0 & $1 / 2$ & 0 & 0 \\
\hline & $\mathrm{N}$ & 0 & $1 / 24$ & $6 / 24$ & 0 & $8 / 24$ & $7 / 24$ & $2 / 24$ \\
\hline & $\mathrm{F}$ & 0 & $1 / 18$ & $4 / 18$ & 0 & $5 / 18$ & $7 / 18$ & $1 / 18$ \\
\hline & $\mathrm{MF}$ & 0 & 0 & $1 / 5$ & 0 & $1 / 5$ & $1 / 5$ & $2 / 5$ \\
\hline
\end{tabular}

\section{Interpretación de algunas probabilidades condicionales de la matriz de transición de 1996 para 1997:}

$P\left[x_{n+1}=1 / X_{n}=1\right]=0$, teniendo en cuenta que este año la temperatura superficial del mar fue Niño Extraordinario, es un hecho imposible que el próximo año la temperatura superficial del mar también sea Niño Extraordinario.

$P\left[x_{n+1}=3 / X_{n}=1\right]=1 / 2=50 \%$, teniendo en cuenta que este año la temperatura superficial del mar fue Niño Extraordinario, hay un $50 \%$ de probabilidad de que el próximo año la temperatura superficial del mar sea de Niño Moderado.

Consecuencia: No habría mucho efecto en cuanto a la economía del país, clima cálido produce un cambio en la fauna marina.

$P\left[x_{n+1}=6 / X_{n}=1\right]=1 / 2=50 \%$, teniendo en cuenta que este año la temperatura superficial del mar fue Niño Extraordinario, hay un $50 \%$ de probabilidad de que el próximo año la temperatura superficial del mar sea Año Frío. 
Consecuencia: Todas las condiciones anómalas van retornando a su normalidad, favoreciendo el retorno de las especies propias de la fauna peruana a ocupar su lugar de origen, la economía se ve favorecida por que encuentra los peces de valor industrial.

$P\left[x_{n+1}=7 / X_{n}=1\right]=0$, teniendo en cuenta que este año la temperatura superficial del mar fue Niño Extraordinario, es un hecho imposible que el próximo año la temperatura superficial del mar sea Muy Frió.

$P\left[x_{n+1}=3 / X_{n}=2\right]=1 / 5=20 \%$, teniendo en cuenta que este año la temperatura superficial del mar fue Niño Fuerte, hay un $20 \%$ de probabilidad de que el próximo año la temperatura superficial del mar sea Niño Moderado.

Consecuencia: El cambio es mas rápido, retorna a la normalidad la pesca tanto industrial como para el consumo directo.

$P\left[x_{n+1}=5 / X_{n}=2\right]=3 / 5=60 \%$, teniendo en cuenta que este año la temperatura superficial del mar fue Niño Fuerte, hay un $60 \%$ de probabilidad de que el próximo año la temperatura superficial del mar sea Año Normal.

Consecuencia: Las condiciones anómalas van retornando a sus condiciones normales.

$P\left[x_{n+1}=1 / X_{n}=3\right]=1 / 15=15 \%$, teniendo en cuenta que este año la temperatura superficial del mar fue Niño Moderado, hay un $15 \%$ de probabilidad de que el próximo año la temperatura superficial del mar sea Niño Extraordinario

Consecuencia: Habría grandes perdidas económicas debido a la poca disponibilidad de las especies marinas y efecto negativos de las fuertes precipitaciones en la parte norte del país.

$P\left[x_{n+1}=6 / X_{n}=6\right]=7 / 18=39 \%$, teniendo en cuenta que este año la temperatura superficial del mar fue Año Frió, hay un $39 \%$ de probabilidad de que el próximo año la temperatura superficial del mar sea Año Frió.

Consecuencia: Hay disminuciones en la temperatura ambiental y favorece a la abundancia de la anchoveta.

$P\left[x_{n+1}=7 / X_{n}=7\right]=2 / 5=40 \%$, teniendo en cuenta que este año la temperatura superficial del mar fue Muy Frió hay un $40 \%$ de probabilidad de que el próximo año la temperatura superficial del mar sea Muy Frió.

Consecuencia: Las condiciones se mantienen iguales. Abundancia de la anchoveta.

\section{Prevenciones}

Efectuar evaluaciones cuantificadas y valorizadas de los efectos negativos (daños) y positivos (beneficios) del fenómeno clasificándolos por sectores económicos y áreas geográficas que permiten orientar a los gobiernos y a los agentes productivos, públicos y privados, en lo que respecta a las acciones de financiamiento para reconstrucción de infraestructura, de reordenamiento de actividades, entre otras.

Diseñar estrategias institucionales que permitan la integración de las entidades nacionales responsables de la investigación, de la previsión, de la evaluación y de la reconstrucción, optimizando un plan de vigilancia de los efectos del fenómeno, desde las señales de alerta hasta las predicciones más confiables para prever los fenómenos adversos y aprovechando al máximo los efectos benéficos dentro del contexto de una adecuada planificación y manejo de zonas costeras y marítimas.

La tabla 1 nos permite observar las diferentes temperaturas de los años 1996 que es considerado año frío (Temperatura observada - valor promedio $=15.9-17=-1.5$ ), y en el año 1997 paso a ser un Niño Extraordinario ( Temperatura observada - valor promedio $=3.46$ ) y 1998 (Temperatura observada - valor promedio $=2.67$ ) fue un Niño Fuerte.

- Se dispone de registros de las temperaturas superficiales marinas en un puerto del norte del país a lo largo del tiempo, sobre los cuales se hace un estudio piloto para realizar predicciones 


\begin{tabular}{|c|c|c|c|c|c|c|c|c|c|c|c|c|c|}
\hline AÑO & ENE & FEB & MAR & ABR & MAY & JUN & JUL & AGO & SET & OCT & NOV & DIC & PROM \\
\hline 1996 & 15,5 & 16,9 & 18,4 & 16,6 & 15,8 & 15,9 & 15,6 & 15,5 & 15,4 & 15,0 & 15,2 & 15,6 & $\mathbf{1 5 , 9 5}$ \\
\hline 1997 & 15,7 & 16,7 & 20,3 & 19,5 & 21,0 & 22,4 & 22,2 & 22,0 & 19,9 & 19,7 & 22,8 & 25,2 & $\mathbf{2 0 , 6 2}$ \\
\hline 1998 & 26,1 & 27,3 & 26,5 & 22,8 & 21,7 & 17,6 & 16,8 & 16,6 & 16,1 & 15,5 & 15,5 & 15,5 & $\mathbf{1 9 , 8 3}$ \\
\hline
\end{tabular}

Tabla 1: Temperaturas Promedio sobre el nivel del mar de 1966, 1997 y 1998 - Chicama (IMARPE)

de la aparición y del posible impacto en la zona del norte de los Andes y de la Amazonia de acuerdo al tipo de fenómeno, mediante el análisis de la cadena de Markov.

- Este estudio piloto brinda la metodología para el análisis en otras zonas costeras ubicadas en la costa central y en la costa sur, observándose el impacto en las zonas transversales correspondientes.

- Sin embargo, existen también impactos positivos tales como la reforestación natural de los desiertos de la costa debido a las abundantes lluvias lo cual ha generado nuevas fuentes de trabajo siendo una inquietud para el futuro aplicar la Investigación Operativa para el aprovechamiento de estas ventajas.

- El presente estudio trata de las diferentes temperaturas promedio superficiales de las aguas marinas registradas en el puerto de Chicama entre los años 1925 y 1996,. Clasificando los diferentes estados y construyendo la matriz de transición de las Cadenas de Markov, para los puertos de Chicama, Paita y Tumbes.

\begin{tabular}{ll}
\hline CLASIFICACIÓN & $\begin{array}{l}\text { TEMPERATURA OBSERVADA } \\
\text {-VALOR PROMEDIO (UT) }\end{array}$ \\
\hline NIÑO EXTRAORDINARIO & $>+2.7^{\circ} \mathrm{C}$ \\
NIÑO FUERTE & $+1.7+2.7^{\circ} \mathrm{C}$ \\
NIÑO MODERADO & $+0.8+1.6^{\circ} \mathrm{C}$ \\
NIÑO DÉBIL & $+0.5+0.7^{\circ} \mathrm{C}$ \\
AÑO NORMAL & $-0.6+0.4^{\circ} \mathrm{C}$ \\
AÑO FRÍO & $\leq-0.7$ \\
AÑO MUY FRÍO & $\leq-1.8$ \\
\hline
\end{tabular}

Tabla 2: Clasificación de la Temperatura superficial del Mar 


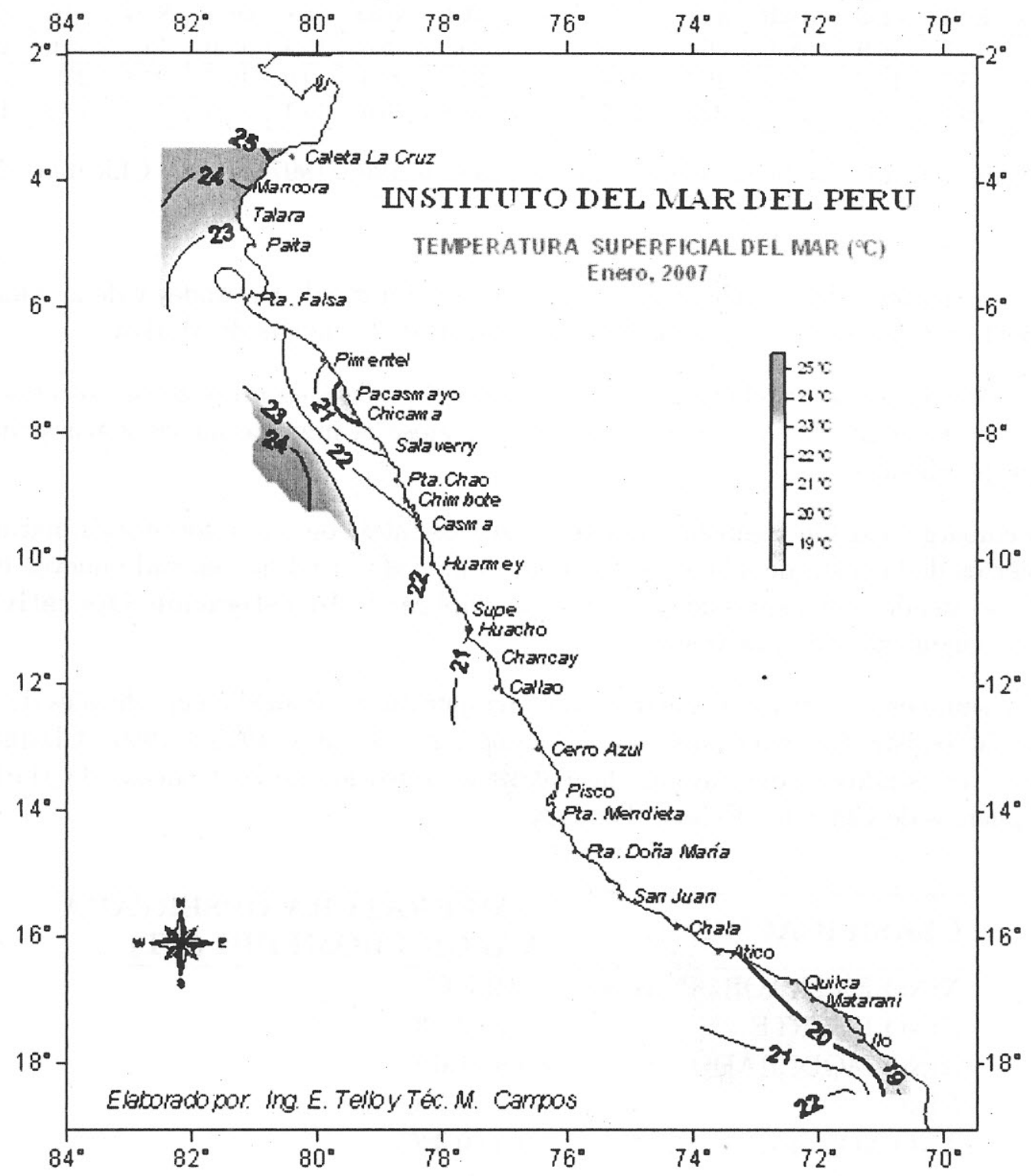

Fig. Temperatura Superficial del Mar $\left({ }^{\circ} \mathrm{C}\right)$ en el mar Peruano durante Enero, 2007

\section{Resultados}

\section{Puerto de Tumbes}

Posición geográfica: $3^{\circ} 28^{\prime}$ S

Espacio Paramétrico - Tumbes

$$
\mathrm{T}=\{1985, \ldots, 2008\}
$$

\section{Interpretación de Tumbes}

Teniendo en cuenta que el año 2008 es clasificado como año normal (Temperatura Esperada Valor Promedio $=26.12-26,30=-0.18$ ) 


$\left.\begin{array}{l|ccccccc} & \text { M. FRÍO } & \text { FRÍO } & \text { NORMAL } & \text { DÉBIL } & \text { MODER. } & \text { FUERTE } & \text { EXTRAO. } \\ \text { M. FRÍO } & 0 & 0 & 0 & 0 & 0 & 0 & 0 \\ \text { FRíO } & 0 & 1 & 3 & 0 & 0 & 0 & 0 \\ \text { NORMAL } & 0 & 2 & 14 & 0 & 0 & 1 & 0 \\ \text { DÉBIL } & 0 & 0 & 0 & 0 & 0 & 0 & 0 \\ \text { MODER. } & 0 & 0 & 0 & 0 & 0 & 0 & 0 \\ \text { FUERTE } & 0 & 0 & 1 & 0 & 0 & 1 & 0 \\ \text { EXTRAO } & 0 & 0 & 0 & 0 & 0 & 0 & 0\end{array}\right)$

Matriz de Probabilidades de Transición - Tumbes

$$
\begin{aligned}
& X_{n+1}
\end{aligned}
$$

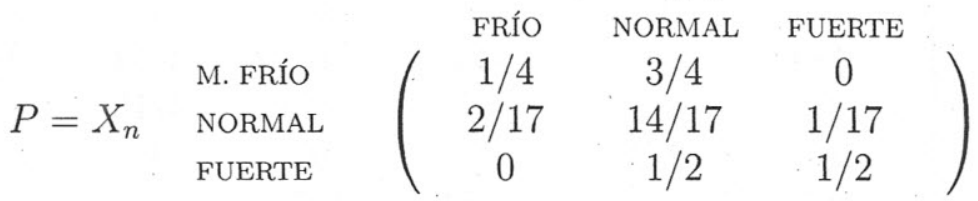

$P\left[X_{n+1}: \operatorname{Normal} / X_{n}:\right.$ Normal $)=82.3 \%$, teniendo en cuenta que este año la temperatura superficial del mar fue Normal, hay un $82.3 \%$ de probabilidad de que el próximo año 2009 la temperatura superficial del mar sea Año Normal.

Consecuencia: Continua la temperatura Normal

$P\left[X_{n+1}:\right.$ Frío $/ X_{n}:$ Normal $)=11.7 \%$, teniendo en cuenta que este año la temperatura superficial del mar fue Normal, hay un $11.7 \%$ de probabilidad de que el próximo año la temperatura superficial del mar sea Año Frío.

Consecuencia: Se produce disminución ligera de la temperatura. Favorable para la anchoveta. $P\left[X_{n+1}\right.$ : Fuerte $/ X_{n}$ : Normal $)=5.8 \%$ teniendo en cuenta que este año la temperatura superficial del mar fue Normal, hay un $5.8 \%$ de probabilidad de que el próximo año la temperatura superficial del mar sea Niño Fuerte.

\section{Consecuencia:}

$P\left[X_{n+1}:\right.$ Fuerte $/ X_{n}$ : Normal $)=6.25 \%$ teniendo en cuenta que este año la temperatura superficial del mar fue Normal, hay un $6.25 \%$ de probabilidad de que el próximo año la temperatura superficial del mar sea Niño Fuerte.

Consecuencia: Se operan cambios un tanto significativos en la ecología marina y en el clima, cercano al normal.

\section{Puerto de Paita}

Posición geográfica: $05^{\circ} 04^{\prime} \mathrm{S}$

Espacio Paramétrico-Paita,

$$
\mathrm{T}=\{1963,1964, \ldots, 2008\}
$$

\section{Interpretación de Paita}

Teniendo en cuenta que el año 2008 es clasificado como Niño Moderado (Temperatura EsperadaValor Promedio: $19.92-18.86=1.05)$

$P\left[X_{n+1}:\right.$ Frío $\left./ X_{n}: N M\right)=20 \%$ teniendo en cuenta que este año la temperatura superficial del mar fue Niño Moderado, hay un $20 \%$ de probabilidad de que el próximo año la temperatura superficial del mar sea Año Muy Frió. 


$$
\begin{aligned}
& \left.\begin{array}{l|ccccccc} 
& \text { MF } & \text { F } & \text { N } & \text { ND } & \text { NM } & \text { NF } & \text { NE } \\
\text { M. FRÍO } & 0 & 0 & 2 & 0 & 0 & 0 & 0 \\
\text { FRÍO } & 1 & 5 & 3 & 0 & 3 & 1 & 1 \\
\text { NORMAL } & 0 & 5 & 7 & 1 & 3 & 0 & 0 \\
\text { DÉBIL } & 0 & 2 & 1 & 0 & 0 & 0 & 0 \\
\text { MODER. } & 1 & 1 & 1 & 1 & 0 & 0 & 1 \\
\text { FUERTE } & 0 & 1 & 1 & 0 & 0 & 1 & 0 \\
\text { EXTRAO. } & 0 & 0 & 1 & 0 & 0 & 1 & 0
\end{array}\right)
\end{aligned}
$$

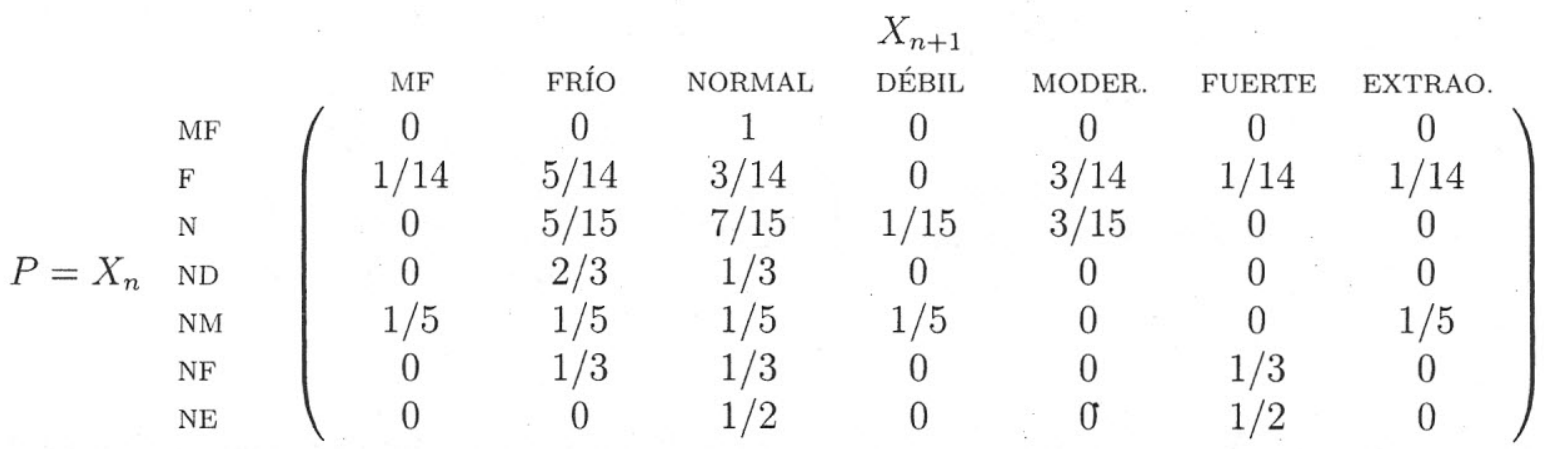

Matriz de Probabilidades de Transición - Paita

Consecuencia: Hay disminución de la temperatura ambiental y favorece a la abundancia de la anchoveta.

$P\left[X_{n+1}:\right.$ Normal $\left./ X_{n}: N M\right)=20 \%$ teniendo en cuenta que este año la temperatura superficial del mar fue Niño Moderado, hay un $20 \%$ de probabilidad de que el próximo año la temperatura superficial del mar sea Año Frió.

\section{Consecuencia:}

$P\left[X_{n+1}:\right.$ Débil $\left./ X_{n}: N M\right)=20 \%$ teniendo en cuenta que este año la temperatura superficial del mar fue Niño Moderado, no existe probabilidad de que el próximo año la temperatura superficial del mar sea Año Normal.

\section{Consecuencia:}

$P\left[X_{n+1}:\right.$ Moderado $\left./ X_{n}: N M\right)=20 \%$ teniendo en cuenta que este año la temperatura superficial del mar fue Niño Moderado, hay un $20 \%$ de probabilidad de que el próximo año la temperatura superficial del mar sea Niño Débil.

Consecuencia: Un ligero incremento en la temperatura ambiental, variaciones ligeras en la ecología marina.

$P\left[X_{n+1}:\right.$ Fuerte $/ X_{n}$ : Frío $)=20 \%$ teniendo en cuenta que este año la temperatura superficial del mar fue Niño Moderado, hay un $20 \%$ de probabilidad de que el próximo año la temperatura superficial del mar sea Niño Extraordinario.

Consecuencia: Se produce cambios pero no significativos sobre todo en la ecología marina, fuertes lluvias (precipitaciones en la parte norte y sequía en la parte sur del país)

\section{Puerto de Chicama}

Posición geográfica $07^{\circ} 43^{\prime} \mathrm{S}$

Espacio Paramétrico-Chicama: $\mathrm{T}=\{1925,1926, \ldots, 2008\}$

Interpretación de Chicama 


$\left.\begin{array}{l|ccccccc} & \text { M.FríO } & \text { FRíO } & \text { NORMAL } & \text { DÉBIL } & \text { MODER. } & \text { FUERTE } & \text { NEXTRAO. } \\ \mathrm{MF} & 0 & 0 & 0 & 0 & 0 & 0 & 0 \\ \mathrm{~F} & 0 & 14 & 8 & 1 & 4 & 1 & 1 \\ \mathrm{~N} & 0 & 9 & 12 & 1 & 5 & 1 & 0 \\ \mathrm{ND} & 0 & 0 & 2 & 0 & 1 & 0 & 0 \\ \mathrm{NM} & 0 & 3 & 5 & 1 & 1 & 3 & 1 \\ \mathrm{NF} & 0 & 1 & 2 & 0 & 3 & 0 & 0 \\ \mathrm{NE} & 0 & 2 & 0 & 0 & 0 & 0 & 1\end{array}\right)$

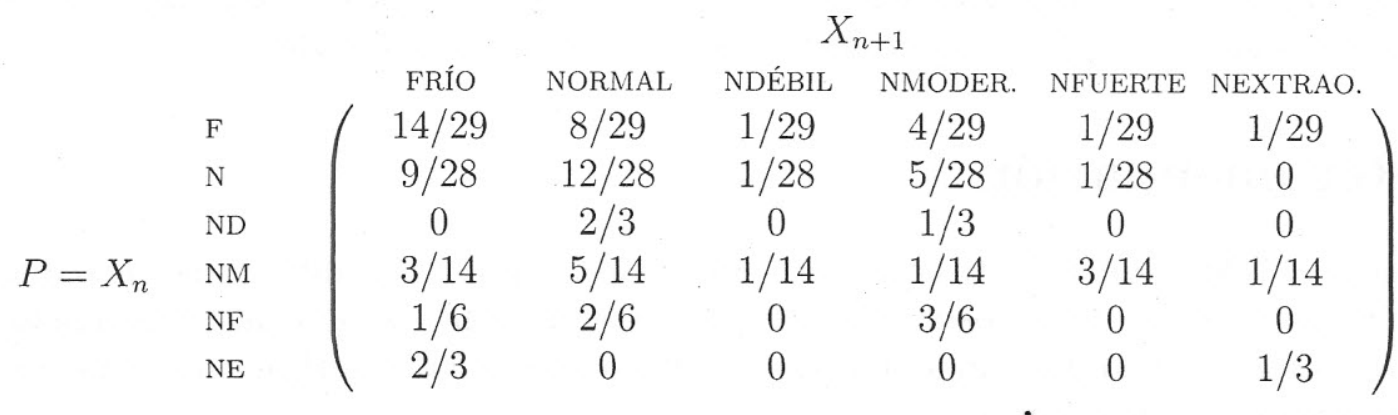

Matriz de Probabilidades de Transición - Paita

Teniendo en cuenta que el año 2008 es clasificado como Año Normal (Temperatura EsperadaValor Promedio : $17.39-17.1=0.27)$

$P\left[X_{n+1}:\right.$ Frío $/ X_{n}:$ Año Normal $)=17.85 \%$ teniendo en cuenta que este año la temperatura superficial del mar fue Año Normal hay un $17.85 \%$ de probabilidad de que el próximo año la temperatura superficial del mar sea Año Frío y propicio para el incremento de la anchoveta.

\section{Consecuencia:}

$P\left[X_{n+1}:\right.$ Normal $/ X_{n}:$ Año Normal $)=42.85 \%$ teniendo en cuenta que este año la temperatura superficial del mar fue Año Normal hay un $42.85 \%$ de probabilidad de que el próximo año la temperatura superficial del mar sea Niño Normal.

Consecuencia: Las condiciones van retomando a sus condiciones normales

$P\left[X_{n+1}\right.$ : Débil $/ X_{n}$ : Año Normal $)=3.57 \%$ teniendo en cuenta que este año la temperatura superficial del mar fue Año Normal hay $3.57 \%$ de probabilidad de que el próximo año la temperatura superficial del mar sea Niño Débil.

\section{Consecuencia:}

$P\left[X_{n+1}:\right.$ Moderado $/ X_{n}:$ Año Normal $)=17.85 \%$ teniendo en cuenta que este año la temperatura superficial del mar fue Año Normal hay un $17.85 \%$ de probabilidad de que el próximo año la temperatura superficial del mar sea Niño Moderado.

Consecuencia: El cambio es mas rápido, retorna a la normalidad la pesca industrial como consumo directo.

$P\left[X_{n+1}:\right.$ Fuerte $/ X_{n}:$ Año Normal $)=3.57 \%$ teniendo en cuenta que este año la temperatura superficial del mar fue Año Normal, hay $3.57 \%$ de probabilidad de que el próximo año la temperatura superficial del mar sea Niño Fuerte.

\section{Conclusiones}

De acuerdo al análisis de las Cadenas de Markov se demuestra que para Tumbes siendo el 2008 un año normal, la probabilidad de que el año 2009 sea normal es del 82.3\%, para Paita siendo el 2008 
un año con Niño Moderado existe la probabilidad del $20 \%$ de que el año 2009 pueda ser: muy frío, frío, normal, niño débil ó Niño extraordinario. En el puerto de Chicama se registra el año 2008 como un año normal y para el 2009 tiene la mayor probabilidad del $42.86 \%$.de que siga siendo normal, pudiendo ser un año frío con una probabilidad del $32.14 \%$. La pesca de la anchoveta es propicia a la abundancia en temperaturas normal, fría y muy fría Las aguas en las que vive la anchoveta debe tener entre 15 y 21 grados centígrados de temperatura y la salinidad debe encontrarse entre los 34,5 y 35,1 UPS. Es decir para que la anchoveta viva y se reproduzca normalmente, debe tener a su disposición alimento como el plankton, aguas con temperatura adecuada y salinidad moderada a la profundidad normal para ellas. En contraposición, por ejemplo, en el año 1983 que fue considerado como un Niño Extraordinario, desde el mes de abril de 1983 hasta abril de 1984 la anchoveta emigró para aguas frías desapareciendo de la zona en estudio.

\section{Recomendación}

El fenómeno El Niño también tiene en el Perú impactos positivos, tales como la reforestación natural de los desiertos de la costa debido a las abundantes lluvias lo cual ha generado nuevas fuentes de trabajo siendo una inquietud para el futuro aplicar la Investigación Operativa para el aprovechamiento de estas ventajas.

Se recomienda realizar campañas para concientizar a la población para no ubicar sus viviendas en lugares de riesgo, cuando se producen las lluvias intensas que origina el fenómeno El Niño y evitar ser afectados por las inundaciones o aluviones.

\section{REFERENCIAS BIBLIOGRÁFICAS}

[1] COLEMAN, R. Procesos Estocásticos. Limusa - Mexico 1976

[2] FELLER, W. Teoría de Probabilidades. Limusa 1973

[3] WINSTON, W Investigación de Operaciones. México 2005

[4] RIVERA, T. Y RIVERA, S Fenómeno del Niño. Lima 1966

[5] Instituto del Mar del Perú (IMARPE) - 2008

[6] Consejo Nacional del Ambiente (CONAM)

[7] CASAVERde .M. Cambios Climáticos - Calentamiento Global . Memoria del Seminario Taller sobre Prevención y Atención de Desastres. Lima. Perú 2004. 